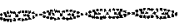

Transaction

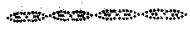

(Received May 21, 1991)

\title{
STRUCTURAL CHANGES OF AMORPHOUS CELLULOSE BY THERMAL AND HYDROTHERMAL TREATMENTS
}

\author{
Akira Isogai, Yusei Akishima, Fumihiko Onabe, and Makoto Usuda
}

Faculty of Agriculture. The University of Tokyo, Bunkyo-ku, Tokyo. 113 Japan

\begin{abstract}
Structural changes of cellulose molecules in non-crystalline region of cellulosic materials by thermal and hydrothermal treatments were studied by using new amorphous cellulose samples, which were prepared from cellulose $/ \mathrm{SO}_{2}$-diethylamine-dimethylsulfoxide solutions. Dry-thermal treatments led to depolymerization of amorphous cellulose and a decrease in moisture contents by forming irreversible hy. drogen bonds, and these changes depended on molecular weight of original anorphous samples, heating temperature, and heating time. However, no crystallization was detected by X-ray diffraction analyses. In the case of thermal treatments of amorphous cellulose samples at $80{ }^{\circ} \mathrm{C}$ and $80 \%$ relative humidity for 1 14 days, no change in amorphous structure, molecular weight, or moisture contents was observed. Hy. drothernal treatments of amorphous cellulose samples in water at temperature higher than $100^{\circ} \mathrm{C}$ brought about crystallization to cellulose $\mathrm{I}$, together with depolymerization and a decrease in moisture contents. These structural changes depended on molecular weight of original amorphous celluloses, heating tempera. ture, and heating time. In comparison with the results of dry-thermal treatments, it was shown that the presence of water remarkably enhanced depolymerization and crystallization to cellulose $\mathrm{V}$.
\end{abstract}

\section{INTRODUCTION}

Cellulosic materials consist of crystalline and noncrystalline regions, and hydrophilic properties of cel. lulosic materials are owing to non-crystalline region. The cellulose I structure in wood does not essentially change during chemical and mechanical pulping. paper making, and other additional processes. On the other hand, cellulose molecules in non-crystalline region may be depolymerized, and may decrease in yields and accessibility during the processes. These microscopic changes in the non-crystalline region must lead to macroscopic changes of cellulose microfibrils and fibers, and furthermore have the influence not only on hydrophilic but also on mechanical properties of cellulosic materials.

Thus, the characterization of non-crystalline region in cellulosic materials is of significance, and many studies concerning thermal, hydrothermal, and pulp. ing treatments of cellulosic materials have been reported from viewpoints of formation of hydrogen bonds and crystallization by using $\mathrm{X}$-ray diffraction analyses, differential scanning calorimetry, infrared spectroscopy, and others [1-9]. In the case of usual cellulosic materials consisting of both crystalline and non-crystalline regions, it is difficult to characterize only the non-crystalline region in the mixture. Therefore, amorphous cellulose models, which were prepared by ball-milling of cellulosic materials or deacetylation of cellulose acetate under nonaqueous and basic conditions, had also been used for characterization [10-17]. These amorphous cellulose samples, however, are unstable even at room temperature under aqueous conditions to form cellulose If to some extent $[10-18]$, whereas the actual non-crystal. line cellulose in cellulosic materials maintains its amorphous structure under these mild aqueous conditions.

On the other hand, we have developed a method of preparing new amorphous cellulose samples, which maintain the amorphous structure even under usual aqueous conditions [19-20]. One of the other features 
of this amorphous cellulose is that the molecular weight can be varied by selecting the original cellulose sample for the preparation of the amorphous cellulose. In this paper, therefore, structural changes of these amorphous cellulose samples were studied to elucidate the structural changes of cellulose molecules in the actual non-crystalline region in cellulosic materials under thermal and hydrothermal conditions.

\section{EXPERIMENTAL}

\subsection{Samples}

Microcrystalline cellulose powder (Cellulose Pow$\operatorname{der}$ A, Advantec Toyo Co. Ltd.), filter pulp (Ashless Filter Pulp, Advantec Toyo Co. Ltd.), linter cellulose, and cotton cellulose were used as the original cellulose samples. Degrees of polymerization (DPv) of the above cellulose samples were determined by the capillary viscosity method using Cuen solutions (cupriethylenediamine hydroxide solution) (22), and were $200,800,1300$, and 1700 .

\subsection{Preparation of amorphous cellulose}

As in previous papers $[19-20]$, regenerated amorphous cellulose samples were prepared from above celluloses by dissolving in the $\mathrm{SO}_{2}$-diethylaminedimethylsulfoxide system and regenerating into water. The regenerated cellulose gel having heterogeneous shape was washed with water several times, and then treated with a blender to form fine gel-particles. The particles thus prepared were washed sufficiently with water, and then freeze-dried. Values of molecular weight of regenerated amorphous cellulose samples were almost equal to those of the original ones.

\subsection{Thermal and Hydrothermal Treatments}

Thermal treatments of amorphous cellulose samples were carried out in an oven at 100,120,140, and $160^{\circ} \mathrm{C}$ under dry conditions. Thermal treatments at high humidity were performed at $80^{\circ} \mathrm{C}$ and $80 \%$ relative humidity for $1-14$ days. After these treatments, the samples were weighed, and then conditioned at 20 ${ }^{\circ} \mathrm{C}$ and $65 \%$ relative humidity for more than 1 day.

In the case of hydrothermal treatments in water, the samples were heated in water in a stainless steel vessel at $100,130,160$, and $190^{\circ} \mathrm{C}$.After this treat. ment, the samples were filtered out, and washed sufficiently with water and finally with acetone. These treated samples were conditioned at $20^{\circ} \mathrm{C}$ and $65 \%$ relative humidity for more than 1 day.

All these thermal and hydrothermal treatments were performed in air.

\subsection{Analyses}

Yields, moisture contents, X-ray diffraction patterns, and DPv of treated samples were measured.

$X$-ray diffraction patterns of pellet samples were recorded on a JEOL JDX-5B diffractometer equipped with a reflection type goniometer, using $\mathrm{Ni}$ filtered $\mathrm{CuK}_{\alpha}$ radiation.

Intrinsic viscosities of cellulose samples were measured using $0.5 \mathrm{~mol}$ Cuen solution and a capillary type viscometer [21]. DPv values were calculated from the intrinsic viscosity, according to the following formula [22].

$$
[\eta]=0.57 \times \mathrm{DPv}^{1.0}
$$

\section{RESULTS AND DISCUSSION}

\subsection{Dry -thermal Treatments}

Fig. 1 shows X-ray diffraction patterns of the orig. inal cellulose samples and the corresponding regenerated ones. All these regenerated celluloses had the amorphous pattern, irrespective of the molecular weight and the crystallinity of the original cellulose samples. Since these amorphous cellulose samples

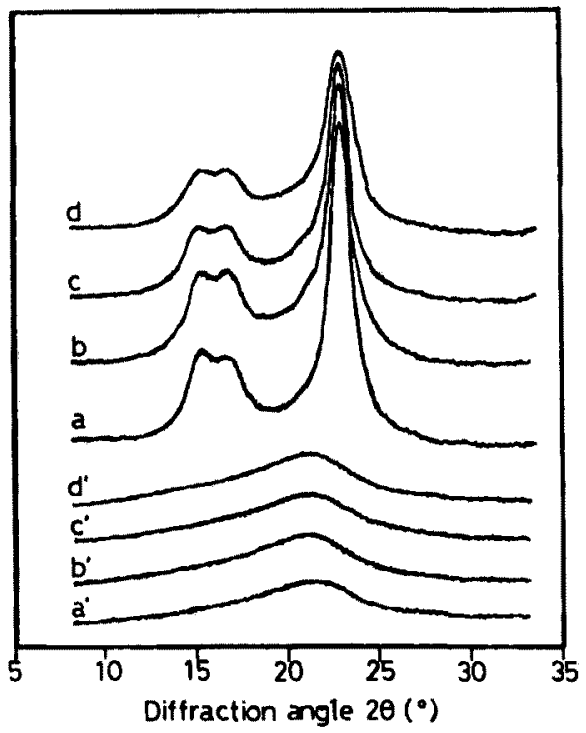

Fig. 1. X-ray diffraction patterns of the original (a-d) and the corresponding regenerated $\left(a^{\prime}-d^{\prime}\right)$ celluloses. $a$; Microcrystalline cellulose powder, b; filter pulp, c: linter cellulose, and d; cotton. 


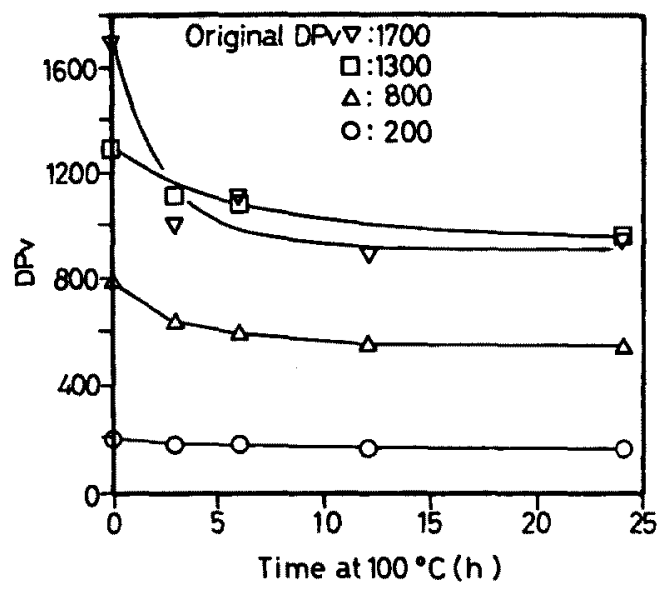

Fig. 2. Degree of polymerization of amorphous cellulose samples subjected to dry-thermal treatments at $100^{\circ} \mathrm{C}$.

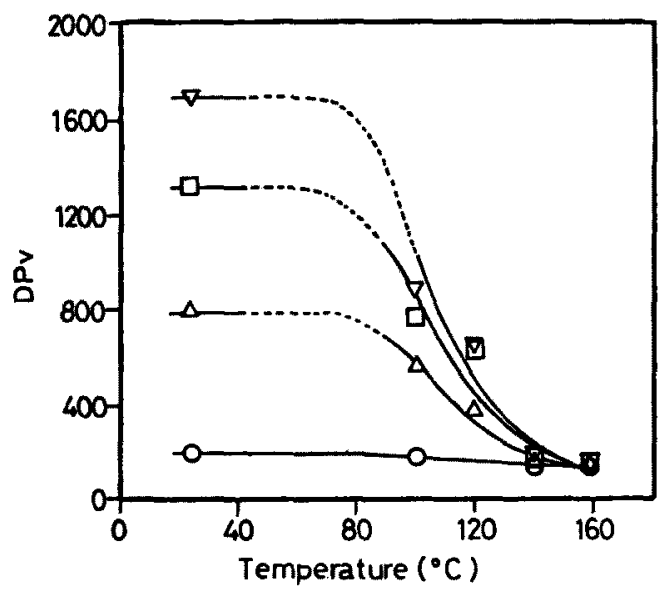

Fig. 3. Degree of polymerization of amorphous cellulose samples subjected to dry-thermal treatments at 100 , 120,140 , and $160^{\circ} \mathrm{C}$ for $12 \mathrm{~h}$.

prepared from microcrystalline cellulose powder, filter pulp, linter cellulose, and cotton had different DPv values, these were named amorphous cellulose $1,2,3$, and 4 , respectively. These four amorphous cellulose samples were treated at $100,120,140$, and $160^{\circ} \mathrm{C}$ for $3,6,12$, and $24 \mathrm{~h}$ in a drying oven.

Fig. 2 shows DPy values of four amorphous cellulose samples treated at $100^{\circ} \mathrm{C}$. Depolymerization at this temperature was negligible even after the treat. ment for $24 \mathrm{~h}$. DPv values of the samples treated at $120^{\circ} \mathrm{C}$ or higher for $12 \mathrm{~h}$ were shown in Fig. 3 . Cellulose molecules in the samples were depolymerized

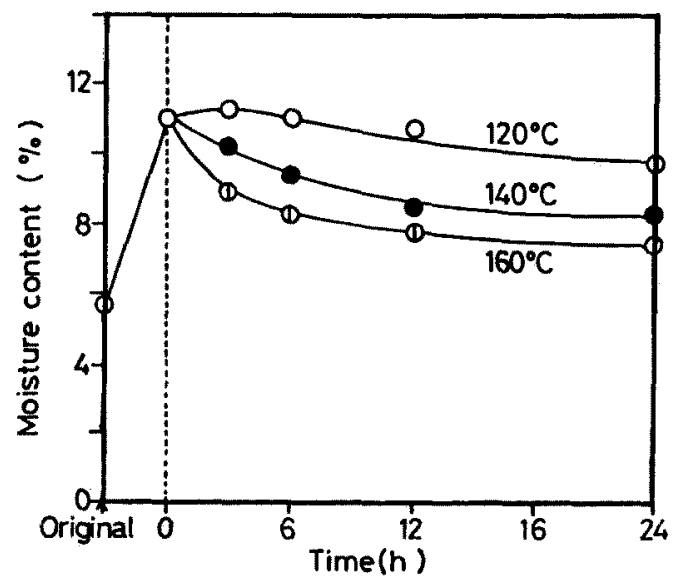

Fig. 4. Moisture contents of amorphous cellulose samples subjected to dry-thermal treatments. Measured after conditioning at $20^{\circ} \mathrm{C}$ and $65 \%$ relative humidity.

as a result of the dry-thermal treatment at $120^{\circ} \mathrm{C}$, and $\mathrm{DPv}$ values decreased with an increase in the heating temperature. Weight-loss of the samples after these treatments was almost negligible for all the samples.

Fig. 4 illustrates the moisture contents of the heated and then conditioned samples, which were prepared from amorphous cellulose 1 . The crystalline cellulose samples used for preparing the amorphous samples had moisture contents of $4.4-5.5 \%$, and the corresponding amorphous cellulose samples had those of $10.9-11.4 \%$. The dry-thermal treatments led to a decrease in moisture contents. Amorphous cellulose 2 , 3 , and 4 also had similar patterns of moisture contents to that of amorphous cellulose 1. The moisture contents of the samples treated at $100{ }^{\circ} \mathrm{C}$ increased at the initial stage, and then decreased. This finding suggests that the accessibility of the amorphous cellulose samples increases at the initial stage of the dry-thermal treatment and then decreases with an increase in the heating time. This phenomenon at the initial stage may not correspond to the thermal behavior of the actual non-crystalline region in cellulosic materials, but may be observed particularly on the amorphous cellulose samples used in this study. However, the trend of the decrease in moisture contents of the model samples, thus observed in this study, indicates that formation of hydrogen bonds, a part of which are stable and uncleaved during conditioning treatments. 


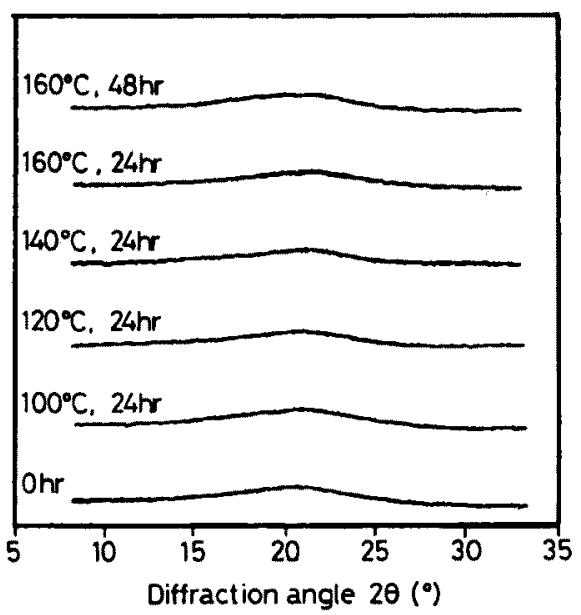

Fig. 5. X-ray diffraction patterns of amorphous cellulose samples subjected to dry-thermal treatments.

proceeds in non-crystalline region under dry-thermal conditions.

X-ray diffraction patterns of the treated samples are shown in Fig. 5 . No crystallization was detected for all the samples and the amorphous structure was maintained, although depolymerization and a decrease in the accessibility took place on the samples under the dry-thermal conditions.

These findings indicate that the structural changes of cellulose molecules in non-crystalline region in cellulosic materials under the dry-thermal conditions are depolymerization and the formation of intra- and/or inter-molecular hydrogen bonds without crystallization. These changes depend on heating temperature and heating time.

The dry-thermal treatments were also performed on wet amorphous cellulose samples, in order to ex amine the structural changes of cellulose molecules in non-crystalline region in cellulosic materials during drying processes from wet state. The temperature in the oven was $105^{\circ} \mathrm{C}$ and the heating time was less than $30 \mathrm{~min}$. Consequently, neither depolymerization nor crystallization took place on cellulose molecules of the samples under these conditions.

\subsection{Thermal Treatments at High Humidity}

Thermal treatments of cellulosic materials under high humidity conditions have been often carried out, associated with accelerated aging of paper [23]. However, even after the treatments at $80^{\circ} \mathrm{C}$ and $80 \%$ relative humidity for $14:$ days, $\mathrm{X}$-ray diffraction analyses showed that all the samples kept the amorphous structure. Furthermore, neither depolymerization nor a decrease in moisture content after conditioning was observed. Thus no structural changes of cellulose molecules in the actual non-crystalline region are plausible to take place under these conditions.

\subsection{Hydrothermal Treatments in Water}

Wood chips undergo hydrothermal treatments dur. ing auto-hydrolysis or prehydrolysis of kraft pulping. During the hydrothermal process, some structural changes including depolymerization may occur on accessible parts such as non-crystalline cellulose and hemicellulose. Then the structural changes of cellulose molecules in non-crystalline cellulose region under these conditions were examined using the amorphous cellulose samples.

Fig. 6 shows DPv values of the samples treated at $100^{\circ} \mathrm{C}$. The amorphous cellulose samples were evidently hydrolyzed even at this temperature in the presence of water, and this phenomenon was different from that observed under the dry-thermal conditions. Furthermore, as shown in Fig. 7, cellulose molecules in the samples were remarkably depolymerized with an increase in heating temperature. Yields after hydrothermal treatments began to decrease at tempera. ture higher than $160^{\circ} \mathrm{C}$ for all the amorphous cellulose samples (Table 1). Fig. 7 and Table 1 show that

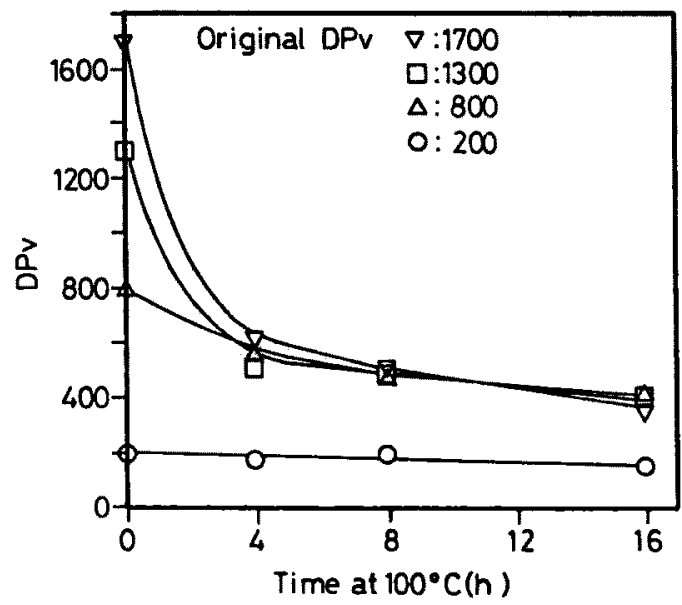

Fig. 6. Degree of polymerization of amorphous cellulose samples subjected to hydrothermal treatments at $100{ }^{\circ} \mathrm{C}$ in water. 
Table 1 Yields after Hydrothermal Treatments of Amorphous Cellulose Samples

\begin{tabular}{lrrrr}
\hline $\begin{array}{l}\text { DPv of } \\
\text { amorphous } \\
\text { cellulose }\end{array}$ & $\begin{array}{r}\text { Time } \\
\text { sample }\end{array}$ & & \multicolumn{3}{c}{ Yield (\%) } \\
\cline { 3 - 5 } & (h) & $130^{\circ} \mathrm{C}$ & $160^{\circ} \mathrm{C}$ & $190^{\circ} \mathrm{C}$ \\
\hline 200 & 2 & 100 & 100 & 77 \\
& 4 & 100 & 98 & 70 \\
& 8 & 100 & 95 & 59 \\
800 & 2 & 100 & 99 & 89 \\
& 4 & 100 & 97 & 56 \\
& 8 & 100 & 97 & 51 \\
1300 & 2 & 100 & 100 & 82 \\
& 4 & 100 & 100 & 80 \\
& 8 & 100 & 100 & 62 \\
1700 & 2 & 100 & 100 & 90 \\
& 4 & 100 & 95 & 69 \\
& 8 & 100 & 94 & 55 \\
\hline
\end{tabular}

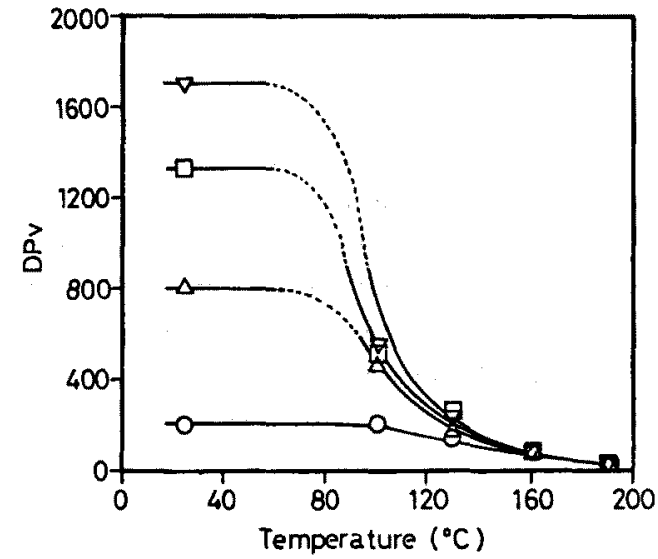

Fig. 7. Degree of polymerization of amorphous cellulose samples subjected to hydrothermal treatments at 100 . 130,160 , and $190^{\circ} \mathrm{C}$ for $8 \mathrm{~h}$ in water.

the yields and DPv values after the hydrothermal treatments at $190^{\circ} \mathrm{C}$ for $8 \mathrm{~h}$ were $51-62 \%$ and $30-$ 40, respectively, for all the samples.

Fig. 8 shows moisture contents of hydrothermally treated and conditioned amorphous cellulose 1 , and the other three amorphous cellulose samples had almost identical patterns of amorphous cellulose 1 . The moisture contents decreased with an increase in the heating temperature and the heating time, and these changes were more remarkable than those for the dry-thermal treatments. These observations indi.

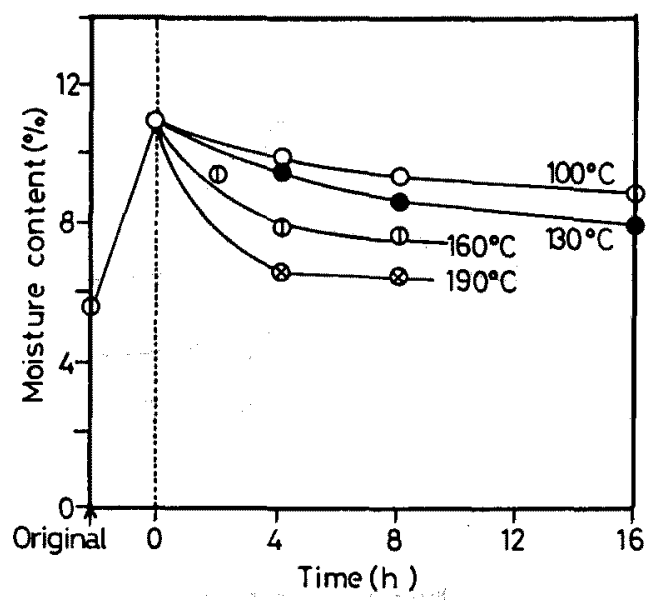

Fig. 8. Moisture contents of amorphous cellulose sam. ples subjected to hydrothermal treatments in water. Measured after conditioning at $20^{\circ} \mathrm{C}$ and $65 \%$ relative humidity.

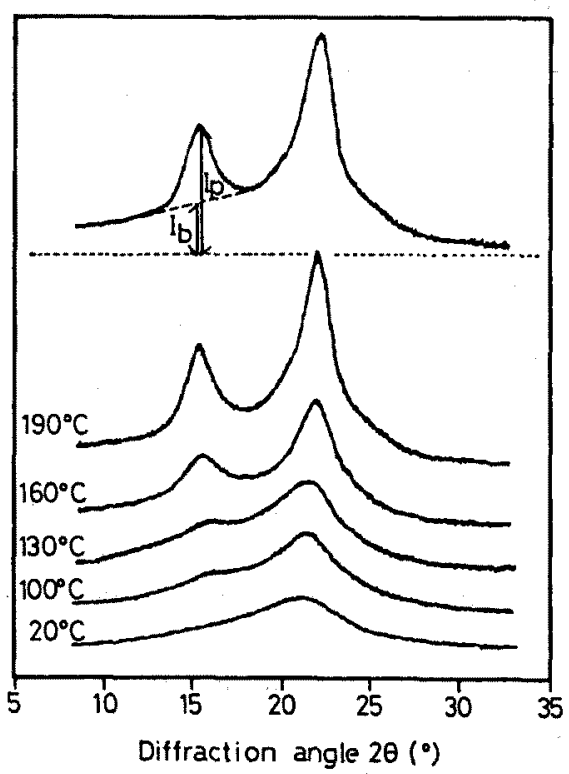

Fig. 9. X-ray diffraction patterns of amorphous cellulose 1 (original DPv $=200$ ) heated in water

cate that the hydrogen bonds, which are stable and uncleaved during conditioning treatments, are formed in non-crystalline region during the thermal treatments.

Fig. 9 and 10 show X-ray diffraction patterns of the treated amorphous cellulose 1 and 4 , respectively. These patterns revealed that the crystallization from 


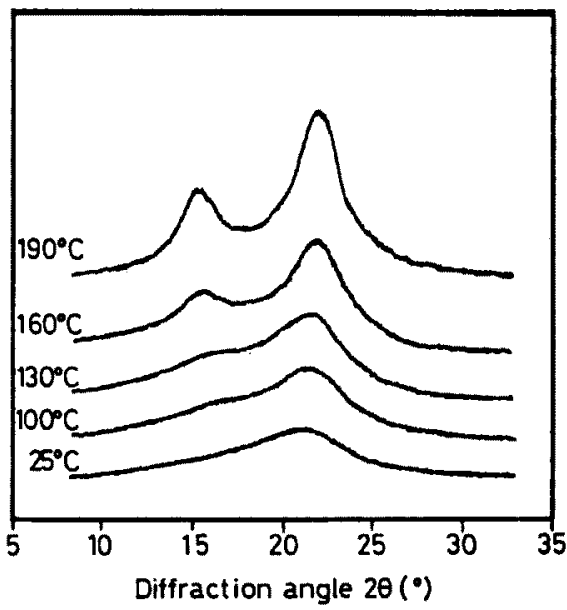

Fig. 10. $X$-ray diffraction patterns of amorphous cellulose 4 (original DPv $=1700$ ) heated in water.

Table 2 Rate Constants to Form Cellulose IV from Amorphous Celluloses by Hydrothermal Treatments

\begin{tabular}{llll}
\hline $\begin{array}{l}\text { DPv of } \\
\text { amorphous } \\
\text { cellulose }\end{array}$ & \multicolumn{2}{c}{$\begin{array}{l}\text { Crystallization rate constants } \\
\text { to cellulose } \mathrm{N}\left(\mathbf{h}^{-\mathbf{l}}\right)\end{array}$} \\
\cline { 2 - 4 } sample & $100^{\circ} \mathrm{C}$ & $130^{\circ} \mathrm{C}$ & $160^{\circ} \mathrm{C}$ \\
\hline 200 & 0.009 & 0.025 & 0.070 \\
800 & 0.006 & 0.020 & 0.050 \\
1300 & 0.006 & 0.020 & 0.043 \\
1700 & 0.006 & 0.020 & 0.048 \\
\hline
\end{tabular}

the amorphous structure to cellulose $\mathrm{N}$ takes place on cellulose molecules in the samples under the hydrothermal conditions. Cellulose IV was slightly de. tected on the samples treated even at $100^{\circ} \mathrm{C}$, and was evidently detected for all the samples treated at 130 ${ }^{\circ} \mathrm{C}$. Thus, in the case of the hydrothermal treatments of the amorphous cellulose samples in water, the clear crystallization from the amorphous structure to cellulose IV proceeds with an increase in the heating temperature and the heating time, and these changes are associated with depolymerization and the decreases in yields and accessibility. Amorphous cellulose samples with higher molecular weight have high er resistance to the structural changes.

The crystallinities of cellulose IV were calculated from the X-ray diffraction patterns using the following formula, according to a modified method of measuring crystallinity of cellulose I and II $[24]$.
Crystallinity of cellulose IV $=\frac{I_{p}-I_{b}}{I_{p}} \times 100(\%)$

Where, $I_{p}$ is the peak intensity at $2 \theta=15.56^{\circ}$ due to $1 \overline{1} 0,110$, and the amorphous region. $I_{b}$ is the intensity of the slope-base-line at $2 \theta=15.56^{\circ}$ due to the amorphous region (Fig. 9). The crystallization to cellulose IV was estimated to proceed according to the pseudo-first-order reaction rate expressions for the treatments at 100,130 , and $160^{\circ} \mathrm{C}$, where the yield loss was negligible. Then the rate constants of the crystallization were obtained from the slopes of the plots, which were illustrated in terms of the logarithms of the crystallinities against the treatment time. The results were shown in Table 2 .

On the basis of these constants, we can estimate the structural changes of cellulose molecules in the actual non-crystalline region in cellulosic materials during these treatments under various conditions. The amorphous cellulose samples used in this work have the properties close to those of the actual non-crystalline cellulose, in terms of the stability of the amorphous structure under aqueous conditions. However, some essential differences in the amorphous structure between regenerated amorphous cellulose samples and the actual non-crystalline cellulose in cellulosic materials may be still present. It is also unknown which amorphous cellulose sample has the most representing molecular weight to cellulose molecules in the actual non-crystalline region. Therefore, further experiments are necessary to solve these problems.

\section{ACKNOWLEDGEMENTS}

This research was supported by a Grant-in-Aid for Science Research (No. 62760133 and 63760133) form the Ministry of Education, Japan.

\section{REFERENCES}

1. T. Hattula, Paperi ja Puu, 68, 847 (1986)

2. T. Hatakeyama, Y. Ikeda, and H. Hatakeyama, Makromol. Chem., 188, 1875 (1987)

3. R. H. Atalla, J. D. Ellis, and L. R Schroeder, J. Wood Chem. T'echnol., 4, 465 (1984)

4. R. H. Atalla and S. C. Nagel, J. Polvm. Sci., Polym. Lett. Ed., 12, 565 (1974)

5. R. H. Atalia and R. E. Whitmore, J. Polym. Sci., Polym. Lett. Ed., 16, 601 (1978) 
6. V. E. Stockmann, Tappi, 54, 2033 (1971)

7. M. Usuda and Y. Matsuoka, J. Japan Wood Res. Soc., 23, 322 (1977)

8. T. Hattula, Paperi ja Puu, 68, 926 (1986)

9. T. Hattula, Paperi ja Puu, 69, 40 (1987)

10. V. M. Gentle, L. R. Schroeder, and R. H. Atalla, ACS Symp. Ser., Washington D.C., No. 340, p. 272 (1987)

11. M. Kimura, T. Hatakeyama, and J. Nakano, $J$. Appl. Polym. Sci., 18, 3069 (1974)

12. H. Hatakeyama and T. Hatakeyama, Sen'i Gakkaishi, 30, T-214 (1974)

13. V. M. Gentle, L. R. Schroeder, and R. H. Atalla, J. Wood Chem. Technol., 6, 1 (1986)

14. H. Hatakeyama, T. Hatakeyama, and J. Nakano, $J$. Appl. Polym. Sci., Appl. Polym. Symp., 28, 743 (1976)

15. H. Hatakeyama, H. Yoshida, and J. Nakano, Car- bohydr. Res., 47, 203 (1976)

16. H. Hatakeyama, T. Hatakeyama, and J. Nakano, Cellulose Chem. Technol., 8, 465 (1974)

17. H. Hatakeyama and $\mathrm{T}$ Hatakeyama, Makromol. Chem., 182, 1655 (1981)

18. I. L. Wadehra and R. St. J. Manley, J. Appl. Polym. Sci. 9, 2627 (1969)

19. A. Isogai and R. H. Atalla, J. Polym. Sci., Polym. Chem. Ed., 29, 113 (1991)

20. A. Isogai and R. H. Atalla, J. Polym. Sci., Polym. Chem. Ed, under contribution

21. TAPPI Standard Methods T230 om-82 (1982)

22. H. Sihtola, B. Kyrklund, L. Laamanen, and I. Palenius, Paperi ja puu, 45, 225 (1963)

23. W. K. Wilson and R. L. Hebert, Tappi, 52, 1523 (1969)

24. A. Isogai and M. Usuda, Sen 'i Gakkaishi, 46, 324 (1990) 